\title{
Cost-effectiveness analysis of the bevacizumab-irinotecan regimen in the treatment of primary glioblastoma multiforme recurrences
}

\author{
DANIEL RUIZ-SÁNCHEZ ${ }^{1,2}$, IRENE IGLESIAS PEINADO ${ }^{2}$, MIGUEL ALAGUERO-CALERO $^{1}$, \\ ALEJANDRO JOSÉ SASTRE-HERES ${ }^{3}$, BENITO GARCÍA DIEZ ${ }^{4}$ and JAIME PEÑA-DÍAZ ${ }^{5}$ \\ ${ }^{1}$ Department of Pharmacy, Central Universitary Hospital of Asturias, 33011 Oviedo, Asturias; ${ }^{2}$ School of Pharmacy, \\ The Complutense University of Madrid, 28040 Madrid; ${ }^{3}$ Department of Pharmacy, San Juan de Dios Hospital, \\ 50006 Zaragoza; ${ }^{4}$ Department of Pharmacy, Severo Ochoa University Hospital, \\ Leganés, 28911 Madrid; ${ }^{5}$ School of Pharmacy, University of Granada, 18011 Granada, Spain
}

Received August 20, 2015; Accepted May 12, 2016

DOI: $10.3892 / \mathrm{ol} .2016 .4871$

\begin{abstract}
The purpose of the present study was to calculate the cost-effectiveness of the inclusion of the bevacizumab $(\mathrm{BVZ})+$ irinotecan $(\mathrm{CPT}-11)$ regimen in the second-line of treatment for primary glioblastoma multiforme. A retrospective cohort study with a control group was performed in which the cost-effectiveness of a course of chemotherapy was calculated based on survival time and the incremental cost between the two lines of treatment. A total of 77 patients were included, 36 of who formed the BVZ/CPT-11 cohort. The median survival time for the non-BVZ control cohort was 13.23 months [95\% confidence interval (CI), 11.79-14.68], while for the BVZ/CPT-11 treatment cohort, the median survival time was 17.63 months (95\% CI, 15.38-19.89). Overall, each year of life gained for each patient treated with BVZ/CPT-11 would cost $€ 46,401.99$. These results demonstrate the effectiveness of the BVZ/CPT-11 combination, but its incremental cost compared with other lines of treatment or the best care available does not appear to be acceptable for public health systems in the current situation of budgetary adjustments.
\end{abstract}

\section{Introduction}

Glioblastoma multiforme (GBM) is a type of cancer that affects the glial cells of the central nervous system (CNS). According to the 2007-2011 report from the Central Brain Tumour Registry of the United States, GBM was the most diagnosed type of primary brain tumour at $45.6 \%$ of the total, and the incidence of GBM was 3.19 per 100,000 inhabitants (1). Furthermore, it is

Correspondence to: Mr. Daniel Ruiz-Sánchez, Department of Pharmacy, Central Universitary Hospital of Asturias, Avenida de Roma s/n, 33011 Oviedo, Asturias, Spain

E-mail: danruizsa@gmail.com

Key words: antiangiogenic, glioblastoma, bevacizumab, irinotecan, cost-effectiveness the most aggressive primary malignant tumour in adults, with a $100 \%$ fatality rate (2).

In Spain, a study performed in Gerona revealed an incidence of 5.88 cases per 100,000 inhabitants/year from 1994-2005 (men, 6.81 cases; women, 4.99 cases) (3). In the region of Asturias (Spain), with a population of 1 million inhabitants, 53,487 tumours were recorded in 2013, of which $152(0.28 \%)$ were tumours affecting the CNS. Within these tumours, GBM exhibited the highest incidence with 38 cases (25.0\%) (4).

The greatest incidence of GBM is found in adults $>45$ years of age (5), and the illness is more prevalent in men than in women, with a male/female ratio of 1.6 (1). Despite numerous scientific advancements, the median survival time for these patients with standard treatment [surgical resection, radiotherapy (RT) and temozolomide (TMZ), followed by 6 cycles of maintenance TMZ] remains low at $~ 14$ months (6).

For cases of recurrence following this first-line treatment, there is presently no approved follow-up treatment in Europe. In May 2009, however, the US Food and Drug Administration did approve the use of BVZ in monotherapy for GBM patients who were diagnosed with progression of the illness and who were previously treated with the first-line standard treatment (7).

The use of bevacizumab + irinotecan (BVZ/CPT-11) in GBM is based on the high vascularisation of this type of tumour and preclinical data showing the dependence of the growth of the glioma on the generation of blood vessels associated with the tumour $(8,9)$. Based on these theoretical premises it appears reasonable to use an anti-vascular endothelial growth factor agent such as BVZ on this type of neoplasm. In studies on GBM, BVZ has been demonstrated to be well tolerated $(10,11)$ and to exhibit antiglioma biological activity (12), with better results than those obtained in historical records (13-27). Furthermore, BVZ appears to induce an improvement in the conservation of neurocognitive function, which suggests a better quality of life (28).

In a previous pharma-economic study, the incremental cost-effectiveness ratio per year of life gained for the first-line treatment with TMZ was considered to be acceptable (29). The present study focuses on the requirement to know the cost-effectiveness of the BVZ/CPT-11 combination, and calculates the incremental cost adjusted to the quality of life that the 
introduction of this second line of GBM treatment supposes, compared with the historical records that are available for patients who have not undergone second-line treatment with this regimen.

\section{Materials and methods}

Study design. A retrospective cohort study with a control group was performed in which the cost-effectiveness of a course of chemotherapy was calculated based on incremental cost. The study was approved by the Ethical Clinical Research Committee of the Principality of Asturias (Oviedo, Spain). Consent for the use of patient data was obtained from the centre management.

Study population. Patients diagnosed with primary GBM between January 2001 and December 2011 in the Principality of Asturias (Spain) and treated in the Central University Hospital of Asturias (HUCA; Oviedo, Spain) were included in this study.

The control cohort included all patients treated with TMZ between January 2001 and December 2006, who were administered the drug by the Department of Pharmacy, HUCA, and fulfilled the inclusion criteria.

The BVZ/CPT-11 cohort included all patients treated with the second-line study regimen between January 2007 and December 2011, and who fulfilled the inclusion criteria.

Inclusion criteria. All patients diagnosed by the primary GBM pathological anatomy, using histological and inmunohistochemistry techniques (30), between January 2001 and December 2011 in the HUCA, who were $>18$ years old and who were subjected to surgical procedures, without differentiating the type of resection and including biopsies, were included in the study.

The control cohort included those patients who received a first-line treatment with RT concomitant with TMZ and/or complete maintenance cycles or treatment until progression, and those who did not receive second-line treatment, or the second-line was distinct to that of the cohort study.

The treatment cohort included all patients who, as well as fulfilling the criteria for the control group with regard to first-line treatment, received second-line treatment with the BVZ/CPT-11 regimen.

Exclusion criteria. All those patients who did not fulfill any of the inclusion criteria and those who lacked any of the data necessary for analysis were excluded.

\section{Definition of variables}

Main variables. Overall survival (OS) was used as a main variable of effectiveness of the study, calculated as the time between the diagnosis and mortality.

Subsequent to calculating the effectiveness of the two cohort treatments, the costs of the second-line treatments were evaluated, assuming that the first-line costs were the same. Using the incremental cost of the study regimen compared with the second-line control cohort schedules, and the median differences in survival time between the cohorts, the present study assessed whether a cost-effective line was involved.
Table I. Costs associated with the treatment.

\begin{tabular}{lrr}
\hline Costs & Price, $€$ & Price, $\$$ \\
\hline Per mg of BVZ & 3.42 & 4.39 \\
Per mg of CPT-11 & 0.10 & 0.12 \\
Per mg of BCNU & 0.64 & 0.78 \\
Preparation (per cycle) & 6.79 & 8.72 \\
Administration (per cycle) & 48.67 & 62.50 \\
Premedication (per cycle) & 1.90 & 2.34 \\
\hline
\end{tabular}

BCNU, carmustine; BVZ, bevacizumab; CPT-11, irinotecan.

Furthermore, a calculation was made of the cost-effectiveness of the BVZ/CPT-11 cohort compared with another 2 subgroups of patients from the control cohort. The first subgroup included only patients who received a second line of treatment and the second included those who received the complete first-line treatment prior to progressing independently of the second line.

For the pharmaceutical-economic study, all of the costs associated with the treatment were analysed (Table I). These costs were divided into four blocks: i) Cost of the antineoplastic drug treatment: The cost per mg was taken into account and complete vials for each patient were not considered. In addition, the acquisition price for the Department of Pharmacy, HUCA, was taken into account, with the resulting economic discounts that this could involve. ii) Cost of the preparation of the antineoplastics in the Department of Pharmacy, HUCA: The costs of the personnel involved in the preparation were calculated (specialist chemist and nursing staff), and the cost of all of the preparations was standardised. The costs of the materials used were disregarded. iii) Costs of administration and monitoring of administration: The cost of the stay in the Outpatient Hospital Service, HUCA. An average $3 \mathrm{~h}$ stay was calculated for each cycle of BVZ/CPT-11 administered, and with the data of the annual cost of this service and the annual number of patients, the costs per hour of stay were obtained for each patient for each cycle administered. iv) Costs of the premedication: The administration of $8 \mathrm{mg}$ ondansetron, $8 \mathrm{mg}$ dexamethasone and $5 \mathrm{mg}$ dexchlorpheniramine was standardised according to the antiemetic protocol of the Department of Oncology, HUCA, for all patients.

After calculating the costs and measuring effectiveness via survival time, the incremental cost-effectiveness ratios per month of life gained (calculated as the median difference of survival) of the BVZ/CPT-11 regimen as a second-line treatment were compared against those of the patients from the control cohort.

Quality of life was evaluated as good where the oncologist decided that the patients could continue to receive the treatment administered. At the time of writing (December 2014), 1 Euro $=0.78$ GBP/1.28 USD.

Secondary variables. The type of second-line treatment received was used as a secondary variable, distinguished by whether it included the BVZ/CPT-11 regimen or not.

Demographic and clinical variables. Age, gender, Karnofsky performance status (KPS) and the type of resection were included. 
Table II. Clinical and demographic characteristics of the patients.

\begin{tabular}{lcc}
\hline Characteristic & BVZ cohort & Control cohort \\
\hline Patients, \% (n) & $46.8(36)$ & $53.2(41)$ \\
Age, years & 53.0 & 56.9 \\
Average & $23-70$ & $27-73$ \\
Range & & \\
Gender, \% (n) & $69.4(25)$ & $51.2(21)$ \\
Men & $30.6(11)$ & $48.8(20)$ \\
Women & 70 & 70 \\
KPS, $\%$ & $60-100$ & $50-100$ \\
Median & & \\
Range & $47.2(17)$ & 0.135 \\
Type of resection, \% (n) & $44.4(16)$ & $46.3(19)$ \\
Total & $8.3(3)$ & $43.9(18)$ \\
Partial & & $7.3(3)$ \\
Biopsy & & $2.4(1)$ \\
N/D & & 0.991 \\
\hline
\end{tabular}

BVZ, bevacizumab; KPS, Karnofsky performance status; N/D, no data.

Statistical analysis. By comparing the differences in OS time and time to progression between the two cohorts, the possible differences in effectiveness were evaluated.

The following analysis was performed using the SPSS 18.0 statistics program for Windows (IBM SPSS, Armonk, NY, USA): Firstly, the two study samples were assessed, calculating typical averages and deviations for the quantitative variables, and the absolute and relative frequencies for the qualitative variables. Overall survival was calculated using the Kaplan Meier method for each study group. $\mathrm{P}<0.05$ was considered to indicate a statistically significant difference.

\section{Results}

In the non-BVZ control cohort, 151 patients were obtained who received TMZ dispensed by the HUCA Pharmacy Service between January 2001 and December 2006. Initially, 25 patients were excluded as they did not have brain tumours. From the rest of the patients treated with TMZ for brain tumours, only 52 were diagnosed with GBM, of which 11 were excluded for being secondary GBM. Finally, there were 41 patients that fulfilled the inclusion criteria.

In the BVZ/CPT-11 cohort, out of the 52 patients diagnosed with a glioma and treated with the study regimen, 11 patients were excluded who were initially diagnosed with low-grade astrocytomas, 3 with oligodendrogliomas and 2 with mixed oligoastrocytomas. Another 3 patients were included with a pathological-anatomical diagnosis of grade III astrocytoma, who were treated as GBM by the oncologist due to doubts in the anatomopathological diagnosis. Finally, there were 36 patients that fulfilled the inclusion criteria

The clinical and demographic characteristics of the patients included in the two cohorts are summarised in Table II. The treatments that the patients received are summarised in Table III.
Table III. First- and second-line treatments received.

\begin{tabular}{lcc}
\hline Treatment & BVZ cohort & Control cohort \\
\hline First line, n/total n & & \\
RT $(60 \mathrm{~Gy})$ & - & $11 / 41$ \\
RT + TMZ $\left(100 \mathrm{mg} / \mathrm{m}^{2}\right)$ & $36 / 36$ & $30 / 41$ \\
TMZ $\left(240 \mathrm{mg} / \mathrm{m}^{2}\right)$ & $34 / 36$ & $34 / 41$ \\
Second line, n/total n & & \\
BVZ/CPT-11 & $36 / 36$ & - \\
CPT-11 & - & $5 / 41$ \\
BCNU & - & $1 / 41$ \\
TMZ & - & $1 / 41$ \\
E.C. & - & $3 / 41$ \\
\hline
\end{tabular}

RT, received radiotherapy (RT) without concomitant temozolomide (TMZ); RT + TMZ, received radiotherapy plus concomitant temozolomide; BVZ/CPT-11, received $7.5 \mathrm{mg} / \mathrm{kg}$ bevacizumab (BVZ) plus $240 \mathrm{mg} / \mathrm{m}^{2}$ irinotecan (CPT-11) every 3 weeks; CPT-11, received $240 \mathrm{mg} / \mathrm{m}^{2}$ irinotecan in monotherapy; TMZ, received $240 \mathrm{mg} / \mathrm{m}^{2}$ temozolomide in second-line treatment; BCNU, received $150 \mathrm{mg} / \mathrm{m}^{2}$ carmustine; E.C., received a clinical trial treatment.

The median survival time was 13.23 months (95\% CI, 11.79-14.68) for the control cohort and 17.63 months (95\% CI, 15.38-19.89) for the BVZ/CPT-11 cohort. This difference in survival was significant $(\mathrm{P}=0.049)$.

When the monitoring period ended, only 1 patient remained alive and continued to receive the second-line BVZ/CPT-11 regimen.

When considering the difference in median survival times between the two cohorts of patients and associating them with the cost of treatment, the incremental cost of survival gained 
Table IV. Separated costs of second-line treatment in the BVZ/CPT-11 cohort.

\begin{tabular}{lcccccc}
\hline Cost & BVZ & CPT-11 & Preparation & Monitoring & Premed & Total \\
\hline Total,$€$ & $599,623.56$ & $9,542.40$ & $2,335.76$ & $16,742.48$ & $1,033.38$ & $629,277.57$ \\
\hline
\end{tabular}

BVZ, bevacizumab; CPT-11, irinotecan; premed, premedication.

Table V. Separated costs of second-line treatment in the control cohort without BVZ/CPT-11.

\begin{tabular}{lrrrrr}
\hline & \multicolumn{5}{c}{ Cost, $€$} \\
\cline { 2 - 6 } Treatment & Treatment & Preparation & Monitoring & Premed & Total \\
\hline CPT-11 & $10,873.40$ & 156.17 & 559.59 & 48.39 & $11,637.55$ \\
BCNU & 647.04 & 0.00 & 0.00 & 0.00 & 647.04 \\
PVC & 13.94 & 13.58 & 48.66 & 0.52 & 76.70 \\
TMZ & $4,410.00$ & 0.00 & 0.00 & 0.00 & $4,410.00$ \\
Total & $15,944.38$ & 169.75 & 608.25 & 48.91 & $16,771.29$ \\
\hline
\end{tabular}

CPT-11, irinotecan; BCNU, carmustine; BVZ, bevacizumab; PVC, procarbazine + vincristine + lomustine; TMZ, temozolomide; premed, premedication.

due to the administration of the second line of treatment can be calculated. All other costs are therefore considered to be irrelevant.

In the cohort with the regimen that included BVZ/CPT-11, the final cost for the 36 patients treated stood at $€ 629,277.57$ (Table IV). The cost in the control cohort was $€ 16,771.29$ (Table V). For all those patients who did not receive a second line within the control cohort, the assigned cost was $€ 0$. The median difference was calculated as follows: Median difference $($ months $)=17.63-13.23$, resulting in 4.4 months of life. The incremental cost of the BVZ/CPT-11 cohort compared with the control cohort was calculated as follows: Incremental cost $(€)=629,277.57-16,771.29$, totalling $€ 612,506.28$.

In this way, increasing survival by 4.4 months for 36 patients came to $€ 612,506.28$, meaning a cost of $€ 46,401.99$ per person for each year of life gained.

\section{Discussion}

As shown in Table II, there were no differences between the patients from either cohort in terms of the clinical and demographic variables; i.e., gender, age, Karnofsky performance status score or the type of resection performed. When directly comparing these variables in the two cohorts, $\mathrm{P}$-values of $>0.05$ were obtained, indicating that there are no significant differences between the groups with regard to these variables.

The characteristics of the present study patients, including the median age, the greater prevalence of men over women and the percentages of the level of resection, were similar to those described in previously published studies by Kreisl et al (median age, 53 years) (12), Friedman et al (median age, 54 years) (10) and Vredenburgh et al (median age, 48 years) (13), and also for the control cohort in the study by Stupp et al (6).
Regarding the mean KPS of the patients at the time of diagnosis, greater fluctuations have been noted. The studies that only evaluated the effectiveness of treatment with BVZ + CPT11 as a second-line therapy included patients with a Karnofsky score of $>70 \%$, and with a range between this value and $100 \%(10,12,13)$. For the present study, in the BVZ/CPT-11 cohort, the KPS range was $60-100 \%$, as patients in a worse general state were included. In the non-BVZ control cohort, the KPS range was 50-100\%. In the study by Stupp et al (6), the patient's general state was measured via the World Health Organisation performance status (31), which measures the general state from 0 to 5 , with 0 being asymptomatic and 5 being mortality, in such a way that the patients included in this study had a range of $0-2$, which was equivalent to a KPS range of $60-100 \%$, similar to the present study.

The present study demonstrated the effectiveness of the BVZ/CPT-11 regimen for the treatment of primary GBM. When comparing the data that were obtained for patients treated solely with RT and concomitant TMZ plus TMZ maintenance, the total median survival time from diagnosis is shown to increase by nearly 4 months, with statistically significant $\mathrm{P}$-value $(\mathrm{P}=0.049)$. The results obtained for the BVZ/CPT-11 cohort in this study are in perfect harmony with those found in the few stage II trials that have been performed thus far. The median overall survival time from the beginning of the second-line BVZ treatment to the date of exitus that was found by Vredenburgh et al (13) was 42 weeks ( 9.6 months) and that measured by Friedman et al (10) was 9.2 months, compared with the 8.8 months in the present study.

A number of factors exist that have been able to affect obtaining these small differences. In the present study, the BVZ/CPT-11 cohort included $11.1 \%$ of patients with a Karnofsky score of $<70 \%$, whereas in certain trials, a KPS score of $\geq 70 \%$ was required as an inclusion criteria (10). The 
percentage of these patients was much lower than in the present study (13), and other characteristics, such as a bilirubin level of $<1.5 \mathrm{mg} / \mathrm{ml}$ or a specific plate count, were not taken into account.

For the non-BVZ cohort data in the present study, the median survival time approached that of the pivotal TMZ trial, where the median survival time was 14 months (2). This discrepancy can be attributed to the fact that the patients in the present study, or at least the majority $(73.2 \%)$, were diagnosed and treated prior to 2005, a date that saw the appearance of the study by Stupp et al (6), which established the basis of treatment with TMZ concomitant with RT and the 6 maintenance cycles. Prior to this time, TMZ was used in fewer cycles; normally 4 cycles of 28 days with 5 successive days of treatment. In addition, there were no cycles of concomitance with RT for the patients who presented a complete resection in the post-surgery scan. The characteristics of the present study patients were also distinct from those of the trial, as inclusion in the latter required adequate renal, hepatic and haematological function, with a series of restrictive values, which was not something that was undertaken when including the present study patients.

Despite all of the aforementioned considerations, an important limitation to the present study was in regard to effectiveness: The survival comparison was not completely correct, as it did not involve totally homogenous cohorts. This is due to the fact that patients included in the second-line BVZ/CPT-11 cohort were evidently patients with better prospects, that is patients in a better general state. In the control patient cohort, we have to suppose that if there are patients with good prospects treated with second-line treatment, there will also be patients with good prospects who for some reason were not treated in the second-line; this could be due to the time, as until the year 2003 no patient was treated with any second-line drug, amongst other factors. Lastly, in this control cohort, there will be patients who progressed to the first line and were not candidates for further lines of treatment, in such a way that there is a bias in the difference in survival time in favour of the BVZ/CPT-11 cohort, which should be taken into consideration.

In addition to the aforementioned bias, another limitation is apparent when comparing the two cohorts, as not all patients received the standard first-line treatment with TM and $\mathrm{RT}$ in the same manner. In the non-BVZ cohort, there were 11 patients who did not receive TMZ concomitant with RT, and later, 7 who did not receive the maintenance treatment with TMZ following the concomitance. In the BVZ/CPT-11 cohort, there were 2 patients who did not receive the RT $+\mathrm{TMZ}$ concomitance and another 2 who did not receive the maintenance. These discrepancies indicate a bias, limiting the criteria of equality that is expected when the survival of the cohorts is compared, in such a way that it is expected that the cohort receiving BVZ/CPT-11 treatment benefits somewhat in the survival comparison.

In terms of costs and cost-effectiveness, the total required to increase survival by 1 year per person equals $>€ 40,000$. When taking into account that the difference in median survival times was not significant and considering all the factors previously mentioned, there appears to be a cost involved that is too high in these current times of budgetary restrictions.
The National Institute for Health and Care Excellence guidelines state the maximum fundable cost at $€ 40,000$, and use the quality-adjusted life years measurement (32). As the present study was retrospective it was impossible to correctly measure quality of life, but it was concluded that the economic cost of increasing survival by 4 months is too high for a public health system with necessarily finite resources, particularly in the current budgetary situation.

The present study data indicate that the second-line BVZ/CPT-11 treatment, although an effective therapy, is not cost-effective, as the incremental cost it supposes compared with other lines of treatment cannot be adopted by the public health system.

\section{References}

1. Ostrom QT, Gittleman H, Liao P, Rouse C, Chen Y, Dowling J, Wolinsky Y, Kruchko C and Barnholtz-Sloan J: CBTRUS statistical report: Primary brain and central nervous system tumors diagnosed in the United States in 2007-2011. Neuro Oncol 16 (Suppl 4): iv1-iv63, 2014.

2. Gurney JG and Kadan-Lottick N: Brain and other central nervous system tumors: Rates, trends and epidemiology. Curr Opin Oncol 13: 160-166, 2001.

3. Fuentes-Raspall R, Vilardell L, Perez-Bueno F, Joly C, Garcia-Gil M, Garcia-Velasco A and Marcos-Gragera R: Population-based incidence and survival of central nervous system (CNS) malignancies in Girona (Spain) 1994-2005. J Neurooncol 101: 117-123, 2011.

4. Tumours register of the health service of the principality of Asturias for 2013. ISSN: 2176-6885. http://www.hca.es/huca/web/main.asp?id_pagina=29. Accessed June 25, 2015.

5. Brem SS, Bierman PJ, Brem H, Butowski N, Chamberlain MC, Chiocca EA, DeAngelis LM, Fenstermaker RA, Friedman A, Gilbert MR, et al: Central nervous system cancers. J Natl Compr Canc Netw 9: 352-400, 2011.

6. Stupp R, Mason WP, van den Bent MJ, Weller M, Fisher B, Taphoorn MJ, Belanger K, Brandes AA, Marosi C, Bogdahn U, et al: Radiotherapy plus concomitant and adjuvant temozolamide for glioblastoma. N Engl J Med 352: 987-996, 2005.

7. Cohen MH, Shen YL, Keegan P and Pazdur R: FDA drug approval summary: Bevacizumab (Avastin) as treatment of recurrent glioblastoma multiforme. Oncologist 14: 1131-1138, 2009.

8. Maxwell M, Naber SP, Wolfe HJ, Hedley-Whyte ET, Galanopoulos T, Neville-Golden J and Antoniades HN: Expression of angiogenic growt factor genes in primary human astrocytomas may contribuye to their growt and progression. Cancer Res 51: 1345-1351, 1991.

9. Takahasi JA, Fukumoto M, Igarashi K, Oda Y, Kikuchi H and Hatanaka M: Correlation of basic fibroblast growt factor expresión levels with the degree of malignancy and vascularity in human gliomas. J Neurosurg 76: 792-798, 1992.

10. Friedman HS, Prados MD, Wen PY, Mikkelsen T, Schiff D, Abrey LE, Yung WK, Paleologos N, Nicholas MK, Jensen R, et al: Bevacizumab alone and in combiantion with irinotecan in recurrent glioblastoma. J Clin Oncol 27: 4733-4740, 2009.

11. Norden AD, Young GS, Setayesh K, Muzikansky A, Klufas R, Ross GL, Ciampa AS, Ebbeling LG, Levy B, Drappatz J, et al: Bevacizumab for recurrent malignant gliomas: Efficacy, toxicity, and patterns of recurrence. Neurology 70: 779-787, 2008.

12. Kreisl TN, Kim L, Moore K, Duic P, Royce C, Stroud I, Garren N, Mackey M, Butman JA, Camphausen K, et al: Phase II trial of single-agent bevacizumab followed by bevacizumab plus irinotecan at tumor progression in recurrent glioblastoma. J Clin Oncol 27: $740-745,2009$.

13. Vredenburgh JJ, Desjardins A, Herndon JE II, Marcello J, Reardon DA, Quinn JA, Quinn JA, Rich JN, Sathornsumetee S, Gururangan S, et al: Bevacizumab plus irinotecan in recurrent glioblastoma multiforme. J Clin Oncol 25: 4722-4729, 2007.

14. Zuniga RM, Torcuator R, Jain R, Anderson J, Doyle T, Ellika S, Schultz $\mathrm{L}$ and Mikkelsen T: Efficacy and patterns of response and recurrence in patines with recurrent high-grade gliomas treated with bevacizumab plus irinotecan. J Neurooncol 91: 329-336, 2009. 
15. Stark-Vance V: Bevacizumab and CPT-11 in the treatment of relapsed malignant glioma. Neuro Oncol 7: 369, 2005 (abstr 342).

16. Raval S, Hwang S and Dorsett L: Bevacizumab and irinotecan in patients with recurrent glioblastoma multiforme (GBM). J Clin Oncol 25 (Suppl): abstract 2078, 2007.

17. Vredenburgh JJ, Desjardins A, Herndon JE II, Dowell JM, Reardon DA, Quinn JA, Rich JN, Sathornsumetee S, Gururangan $\mathrm{S}$, Wagner $\mathrm{M}$, et al: Phase II trial of bevacizumab and irinotecan in recurrent malignant glioma. Clin Cancer Res 13: 1253-1259. 2007.

18. Ali SA, McHayleh WM, Ahmad A, Sehgal R, Braffet M, Rahman M, Bejjani G and Friedland DM: Bevacizumab and irinotecan therapy in glioblastoma multiforme: A series of 13 cases. J Neurosurg 109: 268-272, 2008.

19. Bokstein F, Shpigel S and Blumenthal DT: Treatment with bevacizumab and irinotecan for recurrent high-grade glial tumors. Cancer 112: 2267-2273, 2008.

20. Cloughesy TF, Prados MD, Wen PY, Mikkelsen T, Abrey LE, Schiff D, Yung WK, Maoxia Z, Dimery I and Friedman HS: A phase II, randomized, non-comparative clinical trial of the effect of bevacizumab (BV) alone or in combination with irinotecan (CPT) on 6-month progression free survival (PFS6) in recurrent, treatment refractory glioblastoma (GBM). J Clin Oncol 26 (Suppl): abstract 2010b, 2008.

21. Desjardins A, Reardon DA, Herndon JE II, Marcello J, Quinn JA, Rich JN, Sathornsumetee S, Gururangan S, Sampson J, Bailey L, et al: Bevacizumab plus irinotecan in recurrent WHO grade 3 malignant gliomas. Clin Cancer Res 14: 7068-7073, 2008.

22. Guiu S, Taillibert S, Chinot O, Taillandier L, Honnorat J, Dietrich PY, Maire JP, Guillamo JS, Guiu B, Catry-Thomas I, et al: Bevacizumab/irinotecan. An active treatment for recurrent high grade gliomas: Preliminary results of an ANOCEF multicenter study. Rev Neurol (Paris) 164: 588-594, 2008 (In French).

23. Gilbert MR, Wang M, Aldape K, Lassman A, Sorensen AG, Mikkelson T, Groves M, Werner-Wasik M, Regine W and Mehta M: RTOG 0625: A phase II study of bevacizumab with irinotecan in recurrent glioblastoma (GBM). J Clin Oncol 27 (Suppl): abstract 2011, 2009.
24. Nghiemphu PL, Liu W, Lee Y, Than T, Graham C, Lai A, Green RM, Pope WB, Liau LM, Mischel PS, et al: Bevacizumab and chemotherapy for recurrent glioblastoma: A single-institution experience. Neurology 72: 1217-1222, 2009.

25. Poulsen HS, Grunnet K, Sorensen M, Olsen P, Hasselbalch B, Nelausen K, Kosteljanetz M and Lassen U: Bevacizumab plus irinotecan in the treatment patients with progressive recurrent malignant brain tumours. Acta Oncol 48: 52-58, 2009.

26. Cloughesy T, Vredenburgh JJ, Day B, Das A and Friedman HS Updated safety and survival of patients with relapsed glioblastoma treated with bevacizumab in the BRAIN study. J Clin Oncol 28 (Suppl): abstract 2008, 2010.

27. Ruiz-Sánchez D, Calero MA, Sastre-Heres AJ, García MT, Hernandez MA, Martinez FM and Peña-Díaz J: Effectiveness of the bevacizumab-irinotecan regimen in the treatment of recurrent glioblastomamultiforme: Comparison with other second-line treatments without this regimen. Oncol Lett 4: 1114-1118, 2012.

28. Henriksson R, Asklund T and Poulsen HS: Impact of therapy on quality of life, neurocognitive function and their correlates in glioblastoma multiforme: A review. J Neurooncol 104: 639-646, 2011.

29. Lamers LM, Stupp R, van den Bent MJ, Al MJ, Gorlia T, Wasserfallen JB, Mittmann N, Jin Seung S, Crott R and Uyl-de Groot CA; EORTC 26981/22981 NCI-C CE3 Intergroup Study: Cost-effectiveness of temozolomide for the treatment of newly diagnosed glioblastoma multiforme. A report from the EORTC 26981/22981 NCI-C CE3 intergroup study. Cancer 112: 1337-1344, 2008

30. Louis DN, Ohgaki H, Wiestler OD, Cavenee WK, Burger PC, Jouvet A, Scheithauer BW and Kleihues P: The 2007 WHO classification of tumours of the central nervous system. Acta Neuropathol 114: 97-109, 2007.

31. Oken MM, Creech RH, Tormey DC, Horton J, Davis TE, McFadden ET and Carbone PP: Toxicity and response criteria of the Eastern Cooperative Oncology Group. Am J Clin Oncol 5: 649-655, 1982.

32. McCabeC,Claxton K and Culyer AJ: The NICE cost-effectiveness threshold: What it is and what that means. Pharmacoeconomics 26: 733-744, 2008. 